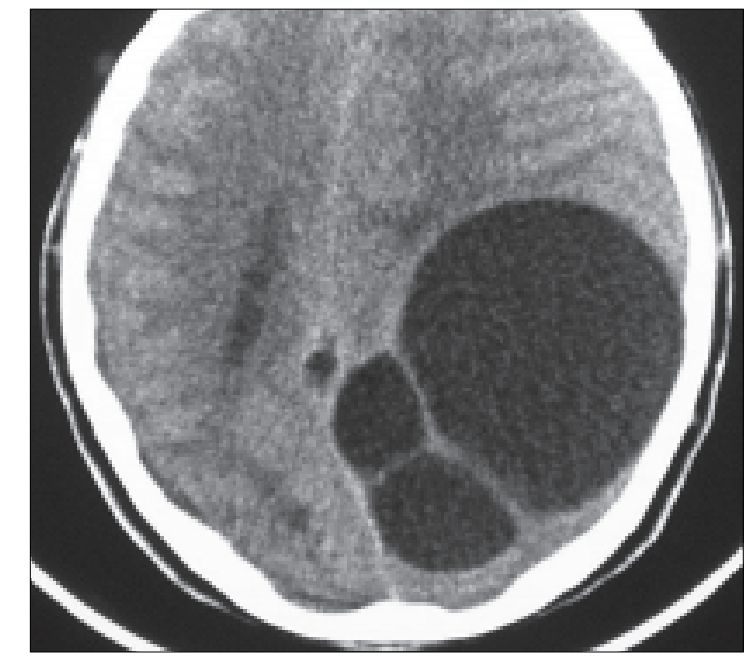

Figure 1: CT scan suggestive of multiple unilobar hypointence cysts with midline shift

\section{Unilobar multiple cerebral hydatid cyst: A rare disease}

\section{Sir,}

A 22-year-old man presented with headache of one year duration. On examination, he had disdiadokokinesia of left side and bilateral papilledema. There were no signs of sensory abnormality/speech disturbances. CT scan of the head showed presence of multiloculated cystic mass in the left parietal lobe with midline shift of $15 \mathrm{~mm}$ toward right side suggestive of multiple hydatid cysts [Figure 1].

His other systemic examinations and investigations for the associated primary or secondary hydatid disease were unremarkable.

The patient was planned for elective surgery but he became unconscious and developed signs of cerebellar

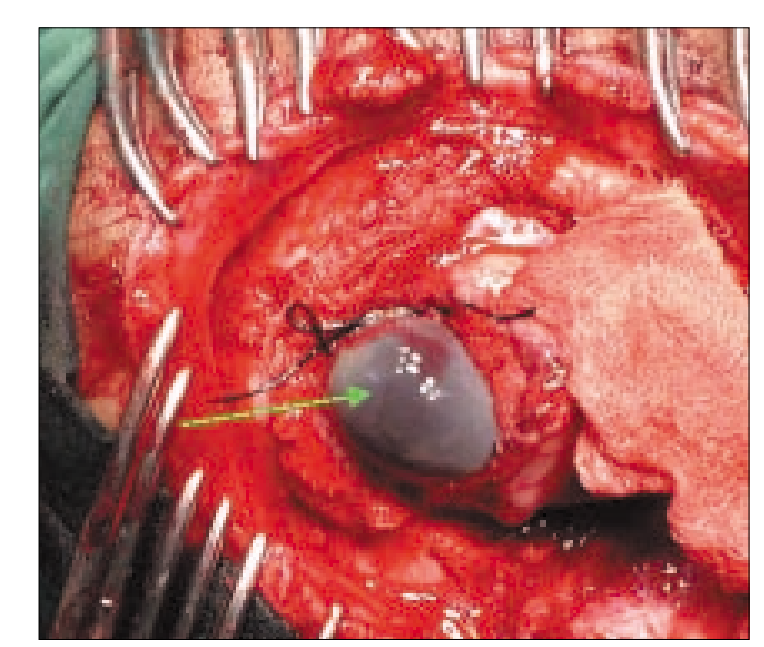

Figure 2: Hydatid cyst seen in the left parietal region of cerebrum

herniation and hence emergency burr-hole was made and the lesion from parietal lobe was aspirated as a desperate measure. The aspirated material showed hydatid cyst membrane. Elective craniotomy was done once the patient became clinically stable (after 24 hours) using left parietal bone flap and the parenchyma over the cysts was gently retracted to expose them [Figure 2]. There was no common ectocyst and all the cysts had separate ecto and endocysts. During surgery we found that, except for the one large cyst that had ruptured because of aspiration, all other cysts were intact. The cysts lay adjacent to each other and there was no common membrane indicating that all the cysts had developed as primary lesions and each of them had separate ecto and endocysts.

Six hydatid cysts were removed from the left parietal lobe parenchyma, taking care not to rupture the cysts, and the resultant cavity was washed for any residual membrane or cyst. 
Postoperative recovery was uneventful. Histopathology of the lesion confirmed it as hydatid cyst. Postoperative therapy consisted of albendazole at $10 \mathrm{mg} / \mathrm{kg}$ dose for a period of six months to minimize the risk of recurrence. The patient is asymptomatic and is doing well since last two years.

Cerebral hydatid cysts constitute only a small percentage of space occupying lesions of the brain more common in children and young adults. ${ }^{[1-3]}$

They are mostly supratentorial and tend to occur in the distribution of the middle cerebral artery. ${ }^{[4]}$

CT scan and magnetic resonance (MR) imaging are the most useful diagnostic techniques. Recently, proton MR spectroscopy using in vivo pyruvate has been described for better confirmation of cystic neoplasms. ${ }^{[5]}$

Surgery is the treatment of choice even in cases of recurrence of the cyst. Aspiration of the hydatid cyst is usually avoided as it can lead to contamination of the other sites and risk of recurrence. Antihelminthic therapy is also given for the treatment of assosciated systemic disease if present. ${ }^{[3]}$

The etiology for the development of the multiple cysts can be either because of the organism like Echinococcus multilocularis or can be because of the rupture of the primary cyst. However, in such cases, there should be common germinal membrane to all the cysts or there should be presence of disease at more than one site.

We found all the cysts without any common membrane making this case unique and hence this presentation was attributed to development of multiple primary unilobar hydatid cysts. ${ }^{[3]}$

Milind P. Joshi, Rahul J. Potode, Anil M. Bhole, Sudhakar R. Joharapurkar

Acharya Vinoba Bhave Rural Hospital Campus, Jawaharlal Nehru Medical College, Sawangi (Meghe) Wardha, India. E-mail:milindj79@yahoo.com

\section{References}

1. Sharma SE, Ray RC. Primary hydatid cyst of the brain in an adult: Report of a case. Neurosurgery 1988;23:374.

2. Atinors N, Senveli E, Donmez T, Bavbek M, Kars Z, Sanli M. Management of Problematic intracranial hydatid cysts. Infection $1995 ; 23: 283-7$.

3. Turget M. Intracranial hydatidosis in Turkey: Its clinical presentation, diagnostic studies, surgical management and outcome: A review of 276 cases. Neurosurg Rev 2001;24:200-8.

4. Yasha TC, Shibu P, Srikanth SG, Shankar SK. 13 year old boy with cerebellar cystic mass. Neurol India 2006;54:100-1.

5. Jayakumar PN, Srikanth SG, Chandrasekhar HS, Kovoor JM, Shankar SK, Anandh B. Pyruvate: An in vivo marker of the cestodal infestation of the human brainon proton MR Spetroscopy. J Magn Reson Imaging $2003 ; 18: 675-80$ 